\title{
Nous nous sommes tant aimés. Retour sur les approches non-formelles de la conception architecturale à l'ère numérique
}

\author{
We loved each other so much. A look back at non-formal \\ approaches to architectural design in the digital age
}

\author{
Daniel Estevez ${ }^{*} 1$ \\ ${ }^{1}$ Laboratoire LRA, Ecole Nationale Supérieure d'Architecture de Toulouse, 31100 Toulouse, France
}

\begin{abstract}
Résumé. L'article propose une analyse épistémologique de certains outils et méthodes de conception en architecture y compris en contexte numérique. Il s'appuie sur des expériences didactiques auxquelles l'auteur a participé sous forme d'ateliers, de workshop, de chantiers etc. Ces recherches sont souvent redevables aux leçons provenant du champ de l'art et à celles qui émanent des recherches pragmatistes qui se sont déroulées dans la dernière partie du XXème siècle. L'article décrit quatre situationstype de conception en architecture et leurs postures de conception sous jacentes. L'hypothèse de cette analyse est que le travail de conception peut être stimulé et déployé à partir de certaines migrations précises que le sujet-concepteur peut accomplir. Passant d'une posture à une autre dans une situation donnée, le concepteur augmente sa puissance d'agir face à des demandes complexes. L'article montre à travers un exemple comment ces migrations, propres aux démarches de conception non-formelle, peuvent parfaitement intégrer les contextes numériques de conception.
\end{abstract}

Mots-clés. SCAN2020, Conception, Architecture, Numérique, Didactique de la Conception.

\begin{abstract}
This paper proposes an epistemological analysis of different tools and design methods in architecture, including those related to digital contexts. It is based on didactic experiences in which the author has participated : workshops, meetings, building events, etc. This research is often fed by lessons coming from the field of art and by those emanating from pragmatist researches that took place in the latter part of the 20th century. The texte describes four typical design situations in architecture and their underlying design postures. The hypothesis of our analysis is that design tasks can be stimulated and deployed on the basis of specific migrations that the subject-designer can accomplish. Moving from one posture to another in a given situation increases the designer's power to act
\end{abstract}

\footnotetext{
*Corresponding author: daniel.estevez@toulouse.archi.fr
} 
in the face of complex demands. Our paper shows through an example how these migrations, specific to non-formal design approaches, can perfectly integrate digital design contexts.

Keywords. SCAN2020, Design, Architecture, Digital Design, Design Didactics

\section{Séparation forme/sens}

La conférence que je vous propose est une sorte d'analyse épistémologique des outils et méthodes de conception en architecture y compris en contexte numérique. Je m'appuie ici sur des expériences didactiques auxquelles j'ai participé sous forme d'ateliers, de workshop, de chantiers etc. qui illustreront cette présentation.

Ces recherches sont souvent redevables aux leçons provenant du champ de l'art. Il y a en effet beaucoup à apprendre pour les architectes à considérer leur activité comme enracinées historiquement dans l'art dont je rappelle que l'étymologie désigne l'action de faire (1165 “ moyen de parvenir à quelque chose, adresse “, 1299 "activité, métier, discipline manuelle ").

Je partirai d'une première leçon un peu paradoxale. Dans le domaine des pratiques artistiques, en effet on considère généralement comme inséparables les notions de forme et de signification. Le terme de "manière" en rend compte de façon assez claire comme on le comprend par exemple dans la phrase de l'artiste Richard Serra lorsqu'il écrit : " c'est la manière dont nous faisons ce que nous faisons qui confère un sens à ce qui est fait ". Ainsi il n'y aurait donc pas d'écart entre l'exécution d'une chose, d'un acte, et puis son contenu sémantique.

Dans le domaine des technologies de l'information cette fusion heurte quelques principes fondamentaux et notamment celui de la disjonction entre information (l'ordre formel, ou syntaxique) et la connaissance (l'ordre sémantique). Je souligne souvent à ce propos la distinction inhérente au numérique qui considère l'information comme un support séparé des connaissances qu'il représente. C'est d'ailleurs la définition officielle de l'informatique comme science : "Science du traitement rationnel notamment par machines automatiques, de l'information considérée comme le support des connaissances humaines ".

On voit donc la nature du problème posé par cette disjonction fondamentale car elle semble incompatible avec la conception architecturale dans sa pratique effective si on la rapproche de celle de l'artiste.

Alors comment l'étude de la conception numérique pourrait-elle assumer cette fusion forme/sens que nous soulignons alors même qu'elle inscrit leur séparation dans sa définition? Historiquement, l'une des voies de résolution de ce paradoxe est constituée par l'analyse des pratiques instrumentées. J'inscris mon intervention dans ce cadre, et je vais évoquer ici des recherches qui s'y inscrivent également le plus souvent. Il s'agit en fait d'adopter une position pragmatiste qui évite autant que possible d'aborder de façon directe les questions de modélisation, de formalisation et a fortiori d'automatisation des pratiques de conception. Les démarches de recherches issues du pragmatisme peuvent donc à ce titre être qualifiées de non-formelle dans la mesure où elles n'intègrent pas la disjonction forme/sens. 


\section{Approches non formelles}

Le qualificatif "non-formel" a été particulièrement utilisé dans le domaine de l'éducation ou il désigne les situations d'apprentissage dans lesquelles celui-ci n'est pas organisé, pas systématique, pas intentionnel et se structure à l'écart d'un cadre formel de formation. Ce type d'apprentissage représente $90 \%$ de l'apprentissage total durant la vie d'une personne y compris d'une personne très scolarisée (cf. le mémoire "Apprendre dans la rue" par Bârbel Paetsch). Un parallèle pourrait être tracé avec l'architecture où le cadre bâti vernaculaire, l'architecture sans architecte, constitue l'essentiel de nos environnements quotidiens.

En matière de conception architecturale et urbaine, j'ai décrit dans un livre de $2015^{\dagger}$ la "Conception Non Formelle" comme un ensemble de procédures et de principes de conception issues des pratiques humaines observables plutôt que de modèles et formalisations théoriques prédéfinies.

Dans l'étude de ces questions non-formelles en architecture, la notion de pratique et d'expérience joue donc un rôle tout à fait central. Du seul fait qu'elle prend l'expérience des situations pour point de départ et non pas une représentation ou une modélisation de ces situations, l'étude de la conception non formelle nous confronte par là à une forme de complexité. Celle-ci est liée au fait de travailler sur l'expérience elle-même plutôt que sur des représentations de l'expérience ou bien alors de considérer toute représentation sous l'angle de l'expérience qu'elle permet.

Il s'agit encore une fois d'un postulat méthodologique pragmatiste. Je voudrais souligner l'importance de cette équivalence entre représentation et expérience pour l'architecte car si celui-ci est bien un praticien en prise avec l'expérience, son action est entourée, équipée, de dispositifs représentationnels.

Parmi ces dispositifs on trouve le dessin sous toutes ses formes : formes tangibles, schématiques, numériques ou analogiques, sous formes de diagrammes ou de traces... Le dessin constitue historiquement bien plus qu'un simple outil représentationnel en matière de conception architecturale dans la mesure où on peut l'utiliser et le faire agir sur le rapport forme/sens selon les usages qu'on décide d'en faire au cours du processus de conception. C'est encore une fois l'art qui peut nous informer sur ce phénomène de la valeur d'expérience du dessin et on peut citer, ici encore Richard Serra, qui écrit à ce propos "le dessin est un moyen de dialoguer avec ce que je fais au moment où je le fais".

La chercheuse Chen Bao dans sa thèse de doctorat intitulée "Écrire l'idée [Xie Yi] : entre l'écriture idéographique et l'écriture architecturale" analyse le fonctionnement linguistique des idéogrammes chinois et son rapport avec le maniement du dessin en architecture. Elle en déduit un principe interculturel de perception-conception propre à l'usage du dessin en conception architecturale ${ }^{\ddagger}$. La perception-conception, selon cette auteure, serait un schème à l'oeuvre aussi bien dans la lecture et l'écriture du dessin que des idéogrammes.

\footnotetext{
$\dagger$ Estevez, D., (2015). Conception Non-Formelle en architecture : Expériences d'apprentissage et pratiques de conception. Eds L'Harmattan, Paris

* Bao, C., (2004). Écrire l'idée, Thèse de doctorat de l’Université Toulouse Jean Jaurès, ED TESC,

https://tel.archives-ouvertes.fr/tel-00736206/file/Bao_Chen.pdf
} 
Quoiqu'il en soit, on voit donc que le dessin a toujours constitué un point d'entrée de choix, en matière de recherche scientifique, pour aborder la conception spatiale, architecturale et urbaine précisément parce qu'il trouble la séparation entre forme et signification.

\section{Postures de conception}

Nous utilisons dans nos recherches la notion de posture, ce terme est emprunté en particulier aux travaux de Madeleine Akrich et Bruno Latour. Pour l'anthropologie des sciences et des techniques le terme posture désigne en effet "l'acteur pris dans une ou un ensemble de relations régies par un unique principe d'équivalence : l'abonné, le spectateur, le contribuable sont autant de termes qui spécifient une posture qui peut être assignée à l'usager des réseaux. Chaque posture est associée à un ensemble de dispositifs techniques mais aussi sociaux" $"$.

Face à ces assignations fonctionnelles et sociales, les usages informels et imprévus des dispositifs techniques peuvent revêtir une certaine importance. Ils renvoient le concepteur à une dimension fondamentale de sa tâche, qui est celle de l'expérimentation comme mode d'appropriation des techniques $^{* *}$. Cela veut dire qu'une posture peut devenir une construction personnelle subjective et non pas un unique déterminisme technique externe.

\section{Situations-type}

Je proposerais ici pour guider mon exposé, de catégoriser les rapports entre les types d'actions menées et les types de faits manipulés au cours des activités de conception par un sujet-concepteur.

Je dois tout d'abord préciser le sens du mot fait dans le cadre de mon intervention. Qu'est-ce qu'un fait? Le pragmatisme définit un fait comme un "élément extérieur au sujet et constitutif d'une situation donnée". Ainsi toute situation d'action est construite comme un ensemble de faits et l'une des tâches principales de la conception consiste à proposer des descriptions de ces faits et à les examiner, les manipuler, les ordonner, les catégoriser etc.

Car pour le pragmatisme de John Dewey il n'existe pas de fait en dehors de sa description, la fusion fait-description est parfaitement assumée dans la vision pragmatiste. C'est pourquoi John Dewey va jusqu'à écrire : "Une signification qui serait complètement abstraite ne pourrait pas être utilisée. Ce sont les connaissances "incorporées" qui sont susceptibles d'être examinée objectivement. Examiner une idée n'est pas une simple façon de parler".

Selon Dewey les faits dépendent donc de leur description représentationnelle pour être opérationnels. Or cette description n'est pas univoque, un fait peut en effet être décrit et redécrit de différentes façons. Redécrire des faits cela revient à les rendre opérationnels, c'est un travail productif et en ce sens c'est une tâche de la conception en architecture.

$\S$ Akrich, M., (1993). Les objets techniques et leurs utilisateurs. De la conception à l'action In Conein, B., Dodier. N., \& Thévenot, L., (eds), Les objets dans l'action. De la maison au laboratoire. Editions de l'EHESS.

** Conesa, J.C., (2009). L'expérimentation comme appropriation du réel In During E., Kihm C., Zabunyan D., In actu-De l'expérimental dans l'art, Les presses du réel. 
J'utiliserai également l'expression "sujet-concepteur", une formule qui est quand à elle tirée en particulier des travaux du chercheur Jean-Charles Lebahar qui situait son travail dans le cadre de la psychologie constructiviste de Jean Piaget. Il définit le sujet concepteur comme : "un sujet psychologique qui conçoit des artefacts dans le domaine de l'architecture et du design industriel, qu'il s'agisse d'une situation opérationnelle ou didactique." [...] "Un SC agit dans une situation de conception définie par les conditions objectives de son activité où interviennent à la fois les caractéristiques du sujet concepteur (connaissances, compétences, expérience, etc.) et celles de la tâche (contraintes techniques et organisationnelles)"

J'ouvre ici une parenthèse. Cette expression (sujet-concepteur) suppose donc une subjectivité à l'oeuvre dans les tâches de conception, une subjectivité qui peut être individuelle mais aussi collective, sociale et même à la limite environnementale c'est à dire une subjectivité intégrant des éléments non-humains. Sur ce dernier point, ("subjectivité intégrant des éléments non-humains") qui peut paraître étrange, on ne souligne pas assez l'apport du chercheur en psychologie cognitive David Perkins sur la cognition distribuée et ce qu'il appelle "l'individu-plus".

Avec la notion de cognition distribuée chez Perkins apparait l'idée que le " milieu physique “ joue un rôle précis dans le développement d'activités reliées à la connaissance et réputées très subjectives comme l'apprentissage ou la conception. Cette distribution physique de la cognition se réalise sur des éléments comme des ordinateurs mais aussi sur toutes sortes d'objets matériels, d'outils et d'instruments de travail. Perkins écrit: "Il est remarquable de constater avec quelle vigueur nous autres êtres humains fonctionnons, si on nous en laisse la chance, comme agents de recrutement pour l'entreprise de la cognition, engageant non seulement d'autres personnes mais aussi les simples objets physiques nous entourant, les arrangeant et les remodelant de manière à en faire des partenaires dans la cognition." $+\dagger$

Je referme la parenthèse et reviens sur les catégories de faits et d'actions à l'oeuvre dans la conception architecturale. Les rapports entre les faits et les actions peuvent être selon moi schématisés autour de quatre situations-type de conception en architecture : le programme, le projet, la convention et l'improvisation. Ces quatre catégories sont établies selon les différents états équilibres qui peuvent exister pour un sujet-concepteur entre la nature des faits qu'il manipule dans les tâches de conception en architecture et la nature des actions qu'il y engage. Deux paramètres sont mobilisés, le degré de précision des faits (niveau de définition) et le degré de détermination des actions (niveau de contrôle), cela définit alors quatre situations types qui induisent dans chaque cas des postures de conception sousjacentes (Figure 1). Je vais les détailler rapidement.

\footnotetext{
${ }^{\dagger}$ Perkins, D.N., (1995). L'individu-plus. Une vision distribuée de la pensée et de l'apprentissage In: Revue française de pédagogie. Volume 111, pp. 57-71.
} 
situations type

programme

projet

convention

improvisation postures induites

ingénieur

praticien

artisan

bricoleur

Figure 1. Situations-type et postures sous-jacentes

\subsection{Situation-type : programme.}

C'est la situation qui nous intéresse aujourd'hui et que je vais analyser plus en détail un peu plus loin. Dans un programme les actions sont déterminées (opérations programmables) et portent sur des faits structurés et formalisés (données). La posture associée à cette situations pourrait être celle de l'ingénieur, c'est à dire la figure d'une épistémologie positiviste.

Pour Claude Levi-Straus l'ingénieur subordonne chacune des tâches qu'il accomplit "à l'obtention de matières premières et d'outils conçus et procurés à la mesure de son projet ". Le profil de l'ingénieur renvoie donc aux méthodes de conception par planification de l'action celle-ci étant établie en fonction de moyens organisés et requis au préalable du projet. L'ingénieur engage une action guidée par une fin et contrôlée par son succès, il se situe donc dans une démarche convergente, déductive et relevant d'une rationalité technique.

\subsection{Situation-type : projet.}

Le projet détermine des stratégies d'action dans lesquelles les actions précises ne sont pas définies a priori contrairement à des opérations programmables et ces actions s'actualisent en se confrontant à une organisation rationnelle et pré-établie des faits manipulés. Cette organisation préalable est souvent décrite comme une structure.

En architecture, cette organisation résulte souvent d'un travail de catégorisation qui produit une structure de faits possible pour la situation abordée. C'est notamment le cas des conceptions basée sur le schéma de l'enquête, la collecte et l'interprétation productive plutôt que la modélisation. Je fais référence ici aux travaux récents de la chercheuse 
Tiphaine Abénia sur les grandes structures abandonnées ${ }^{*}$. Elle y décrit des liens précis entre catégorisation et création d'analogies entre échantillons (ou faits). Toute catégorisation repose sur une stratégie interprétative de la réalité. Catégoriser signifie mettre en oeuvre un processus inductif d'organisation des faits, de production d'une structure de faits. Ce processus peut s'apparenter à une méthode de conception et non pas une simple activité analytique. Toutes les approches dites structuralistes de la conception (Hertzberger, Fuller) considèrent que l'objet du travail de conception n'est pas la production d'un objet mais la création d'une structure.

Cette réflexion est conforme encore une fois aux postulats des approches pragmatistes de John Dewey lorsqu'il affirme que l'enquêteur "organise les faits dans l'observation", c'est à dire comme l'indique Tiphaine Abénia à partir de son travail d'interprétation/catégorisation.

Le rapport entre situation-projet et action stratégique du sujet-concepteur a été illustré par une métaphore que livre Edgar Morin en décrivant un adolescent qui dévale un sentier de montagne. Le sentier constitue une structure ordonnée possédant des capacités spécifiques mais la course est une performance qui repose sur une stratégie d'usage de ces capacités. Il va plus loin en livrant l'analyse suivante : “La stratégie suppose l'aptitude du sujet à utiliser, pour l'action, les déterminismes, les collaborations et les aléas extérieurs, on peut la définir comme la méthode d'action propre à un sujet en situation de jeu où, pour accomplir ses fins, il s'efforce de subir au minimum et d'utiliser au maximum les contraintes, les incertitudes et les hasards de ce jeu. "§§

Pour de nombreux auteurs, cette situation de projet induit une posture spécifique, celle du "praticien-réflexif" décrit par le chercheur Donald Shön dans son ouvrage éponyme***. Le praticien-réflexif désigne la figure d'un sujet en action d'interprétation et de critique dans un contexte d'incertitude et d'instabilité. Cette image insiste donc sur les compétences du concepteur en matière d'appréciation des situations en cours de route et de stratégie d'action. Elle respecte la métaphore du coureur sur le sentier de montagne mais aussi celle du projet in situ, modalité pédagogique que nous avons souvent expérimentée. Selon Donald A Schön, cette réflexion-intégrée-à-l'action constitue un processus d'interaction continue avec le contexte. Schön analyse ce processus selon une autre métaphore, celle d'une "conversation réflexive avec la situation".

Il s'agit d'une vision transactionelle de la posture de conception en situation de projet. Une vision dans laquelle le concepteur façonne la situation mais est également façonné par elle dans son interprétation. (Ce qui nous renvoie à certains postulats de la cognition distribuée)

\subsection{Situation-type : convention.}

La convention (la situation conventionnelle) repose sur des actions déterminées (des règles tacites, des habitudes, des usages convenus, de bonnes pratiques) et elle concerne des faits non décrits (des faits implicites). La posture induite ici serait celle de l'artisan.

†t Abenia, T., (2019). Architecture potentielle de la Grande Structure Abandonnée. Catégorisation et projection. Thèse de doctorat, Université de Toulouse Jean Jaurès, ED TESC.

$\S \S$ Morin, E., (1986). La Méthode 3. La connaissance de la connaissance, Le Seuil, Paris, p.62

*** Schön D.A., (1994). Le praticien réflexif : à la recherche du savoir caché dans l'agir professionnel, Montréal

: Logiques. (Coll. Formation des maîtres) 
L'artisan est celui qui travaille la chose en soi, il produit des artefacts mais surtout produit une augmentation de son savoir-faire, son action débouche donc sur un savoir constitué (une praxis) que définit notamment le terme " métier ". Pour Richard Sennett, "le métier désigne un élan humain élémentaire et durable, le désir de bien faire son travail en soi. Il va bien plus loin que le travail manuel qualifié ; il sert le programmeur d'ordinateur, le médecin et l'artiste. Dans tous ces domaines, le métier se focalise sur des normes objectives, sur la chose en soi."

L'image du concepteur comme artisan renvoie aussi à l'idée d'un savoir-intégré-àl'action. La notion de savoir tacite, (ou savoir caché) que décrivent des auteurs comme Michael Polyiani, Jean Piaget etc. semble être une des caractéristiques des situations conventionnelles de conception. C'est pourquoi nous pourrions dire que les contextes de production conventionnels dans lesquels il n'est pas nécessaire d'expliciter formellement les connaissances pour agir, collaborer et produire favorisent la posture de l'artisan.

\subsection{Situation-type : improvisation.}

L'improvisation repose sur des tactiques dont les actions se déterminent au cas par cas selon des faits qui ne sont pas structurés a priori. L'improvisation possède donc un caractère fondamentalement contextualiste et interprétatif c'est à dire qu'elle favorise la capacité d'appréciation du moment et de l'occasion d'agir. Cette situation-type induit la posture du bricoleur.

Le bricoleur est celui qui est capable d'exécuter un grand nombre de tâches diversifiées sans programme préalable et surtout sans outil dédié à une fonction spécifique du programme. Levi-Straus affirme que l'univers instrumental du bricoleur est clos et la règle de son jeu est de : " toujours s'arranger avec les moyens $d u$ bord, c'est-à-dire un ensemble à chaque instant fini d'outils et de matériaux, hétéroclites au surplus, parce que la composition de l'ensemble n'est pas en rapport avec le projet du moment mais est le résultat contingent de toutes les occasions qui se sont présentées d'enrichir le stock [de ses outils] avec les résidus de constructions et de destructions antérieures."

L'image du concepteur comme bricoleur renvoie donc à l'idée d'indétermination de toute production novatrice. Il s'agit d'une situation que nous avons eu l'occasion d'expérimenter lors de sessions de chantiers de conception in situ ${ }^{\S \S}$. On peut préciser, que l'improvisation que le bricoleur engage n'est pas exactement celle du praticien-réflexif dans sa "conversation avec la situation" que nous venons d'énoncer. Elle est plus ouverte et moins structurée.

Je citerais les travaux de l'architecte et chercheur Christophe Hutinqui a exploré cette situation d'improvisation dans plusieurs de ses projets d'architecture. Avec par exemple son projet structuraliste de lotissement vertical Haut-Plateaux à Bègles (France) dans lequel les projets installés sur l'infrastructure du bâtiment ne sont pas programmés par le concepteur mais définis par les habitants eux-mêmes à partir des capacités définies par la construction, les réseaux, l'orientation etc.

†† Sennett R., (2010). Ce que sait la main, la culture de l'artisanat, Albin Michel, Paris, p.20

$+1+$ Levi-Straus, C., (1960). La Pensée sauvage, Paris, Ed. Plon, Paris. p 27

$\S \S \S$ Estevez, D. \& Hutin, C. (2010), blog d'archives http://learning-from.over-blog.fr/ (consulté 2020) 
La même démarche d'improvisation est explorée dans son projet des extensions en façade pour les immeubles de logement du Grand Parc à Bordeaux. Dans ce travail des volumes construits mais indéterminés dans leurs fonctions sont ajoutés aux appartements existants (outils a-fonctionnels de Levi-Straus) et par la suite ces volumes sont appropriés par les habitants selon leurs intentions a posteriori.

\section{migrations}

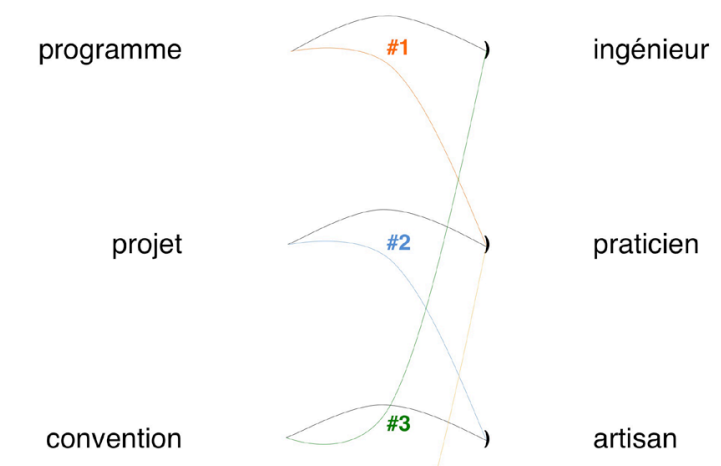

improvisation

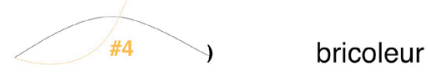

Figure 2. Situations-type et migrations de posture (flèches couleurs)

L'hypothèse que je voudrais exposer dans cette conférence est que le travail de conception en architecture peut être stimulé et déployé à partir de certaines migrations $^{* * * *}$ précises que le sujet-concepteur peut accomplir. Passant d'une posture à une autre dans une situation donnée, le concepteur augmente sa puissance d'agir face à des demandes complexes. Il s'agit pour le sujet-concepteur de faire l'expérience d'une posture défavorable à la situation donnée. Nous avons examiné plusieurs de ces migrations "élémentaires" (Figure 2.) celle du praticien en situation-type programme (\#1), de celle de l'artisan en situation projet (\#2), de celle de l'ingénieur en situation conventionnelle (\#3) de celle du praticien réflexif en situation d'improvisation (\#4)

Il ne s'agit donc pas de promouvoir une posture de conception unique, posture considérée comme supérieure vis à vis des autres en fonction de la situation-type abordée. $\mathrm{Au}$ contraire, nous considérerons que les autres postures ne sont pas non plus réputées inappropriées pour la même situation-type.

Je veux enfin préciser que mon exposé s'appuie sur des terrains d'expérimentations essentiellement didactiques prenant souvent la forme de workshop in situ. Je vais ici me limiter à décrire et argumenter à titre d'exemple uniquement un seul cas de migration dans une seule situations-type, celle du programme. 


\title{
5. Situation-type \#1 (le programme)
}

\author{
migrations \#1 \\ programme

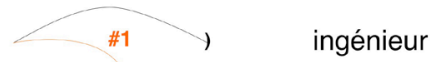 \\ , praticien
}

Figure 3. De l'ingénieur au praticien, exemple de migration de posture.

Pour permettre de comprendre la première migration décrite dans ce schéma (posture praticien en situation programme, Figure 3.), il faut revenir sur quelques caractéristiques de la situation-type programme et sur sa posture induite.

Dans cette situation comme je l'ai dit, la conception est en relation avec le schéma général du programme. Il est bien clair que cette situation touche souvent le contexte numérique mais pas uniquement et pas systématiquement. Un programme, c'est un système d'opérations déterminées portant sur des faits structurés (des informations). Le modèle programme/données s'inscrit dans une approche hypothético-déductive de la conception.

J'ai évoqué la posture de l'ingénieur comme posture induite dans cette situation-type. Dans sa thèse de doctorat sur la conception par bricolage ${ }^{\dagger \dagger \dagger \dagger}$ en architecture Fanny Léglise rappelle que chez Levi-Straus "le modèle de l'ingénieur fait référence à l'homme cartésien maître et possesseur de la nature, séparé de la matière sensible par la conscience qui lui permettrait d'y appliquer des formes intelligibles."

C'est pourquoi l'ingénieur définit son projet au préalable, le planifie et ne se laisse pas guider par un stock de moyens disponibles. Au contraire, il exige de disposer des moyens de l'action qu'il programme et cela bien entendu préalablement à la conduite de l'action. L'ingénieur hiérarchise ses connaissances théoriques et pratiques, et attribue des fonctions précises à ses moyens techniques pour les rendre opérationnels (mais dans le même temps écrit Fanny Léglise il restreint "le champ des solutions qu'il pourra apporter à un problème.”)

En 1964, avec son livre "Notes on the synthesis of form", l'architecte Christopher Alexander fut probablement l'un des premiers architectes à tenter d'intégrer la structure informationnelle données/programme dans la conception architecturale. La conception qu'il modélise est un processus formel fondée sur le couple analyse et synthèse qui s'organisent systématiquement selon une structure en arbre de données. Le schéma hiérarchique de décomposition cartésienne est appliqué ici dans toute ses possibilités. Il écrit : "le point de départ de départ de l'analyse ce sont les données, le produit final de l'analyse est le programme qui est un arbre hiérarchique de données. Le point de départ de la synthèse est

$\dagger \dagger \dagger$ Léglise, F., (2020). La conception par bricolage comme art de la situation. Architecture, urbanisme, design. Thèse de doctorat, Université de Toulouse Jean Jaurès, ED TESC. 
le diagramme. La produit final de la synthèse est la réalisation du problème qui est une arborescence de diagrammes."

En assumant la séparation entre information et signification dans son modèle de conception, Alexander assume donc l'hypothèse opérationnelle fondamentale de la théorie de l'information.

Ses premières recherches, subissent l'influence de celles de Noam Chomsky à la même époque, et s'inscrivent dans le projet général, explicite ou non, d'une l'intelligence artificielle substitutive. Il s'agit précisément de formaliser de façon syntaxique des éléments sémantiques. Cette modélisation repose elle-même en grande partie sur des schémas hiérarchiques, des arbres. Noam Chomsky propose une linguistique générative fondée sur des règles préalables et des transformations productives. Avec ce schéma "descendant", la grammaire de Chomsky a pour objet de générer tous les énoncés possibles dans une langue c'est pourquoi on la nomme générative. C'est également la raison pour laquelle cette grammaire a exercé une certaine fascination dans d'autres disciplines (en architecture notamment) qui pouvaient envisager l'idée d'une automatisation des processus de conception, de décision ou d'expression.

On cherche donc en quelque sorte à procéder à une "sémantisation" automatique de l'information, à déduire la signification à partir de la forme mais aussi en sens inverse à générer de la signification à partir de la forme. En fait, cela suppose de modéliser et d'automatiser les processus interprétatifs (qui relèvent normalement des sujets humains) en les formalisant dans un cadre logique (hypothético-déductif).

Dans les années 1970 les travaux de William J. Mitchell sont particulièrement représentatifs des approches déductives radicales appliquées à la conception architecturale. Ces recherches prendront par exemple pour terrain le cas des plans de villa de l'architecte Andréa Palladio. Ici la visée productive est très claire, il s'agit d'automatiser la génération de propositions architecturales (des plans de villas) à partir de règles formelles préalables. Le domaine d'application de ces formules est réduit à un terrain d'étude circonscrit, censé être plus facile à maîtriser ou à modéliser. La syntaxe générative des plans palladiens est censée produire de nouveaux plans inédits, des plans qui auraient pu être conçus par Palladio ${ }^{\S \S \S}$.

Ces modèles totalisants, substitutifs du sujet-concepteur et fondés sur des structures d'arbre, n'ont pas connu d'aboutissement opératoires directs en architecture. Même s'ils ont eu une certaine influence notamment dans l'enseignement et marqué toute une partie des pédagogies de la conception architecturale à l'époque. En réalité l'échec des diverses tentatives d'automatisation de la conception architecturale était inhérent aux présupposés de cette démarche, comme l'avait montré le philosophe de la technique Gilbert Simondon car selon lui le principe d'automatisme ne peut conduire qu'à la production de machines fermées. Des machines fermées c'est à dire déterminées et inaccessibles à des usages non programmés.

En revanche, ce que Simondon appelle les machines ouvertes sont au contraire des systèmes faisant place à des marges d'indétermination. Il oppose clairement automatisme et indétermination. Pour lui l'automatisme peut conduire à la réduction des capacités d'usage

Htt Alexander C., (1964). Notes on the synthesis of form, Oxford UP, p.84

$\S \S \S \S$ Benros D., Duarte J., \& Sean H., (2012). A New Palladian Shape Grammar A Subdivision Grammar as alternative to the Palladian Grammar In International Journal of Architectural Computing, December 2012 
des objets techniques, c'est à dire à leur fermeture. Une machine ouverte est caractérisée au contraire par son indétermination. Il écrit : "le véritable perfectionnement des machines, celui dont on peut dire qu'il élève le degré de technicité, correspond non pas à un accroissement de l'automatisme, mais au contraire au fait que le fonctionnement d'une machine recèle une certaine marge d'indétermination. “******

Ainsi pour que la machine à dessin contemporaine, le logiciel graphique notamment, soit une machine ouverte comment faire ? Si l'on suit Simondon, il faudrait simplement maintenir le principe d'automatisme à un niveau précis par lequel il augmente la capacité d'usage du concepteur au lieu de la diminuer et de la refermer. Il faut parvenir à un équilibre par lequel la machine logicielle devient incitative pour l'utilisateur. (Une machine ouverte ou encore une machine incitative est un dispositif qui peut jouer un rôle stimulant et non pas substituant pour le sujet concepteur.)

\section{Migration \#1 (l'ingénieur inductif)}

Nous pouvons aborder à présent la première migration de posture, celle qui cherche à rendre possible la posture du praticien-réflexif capable d'avoir un usage ouvert de ses outils tout en se maintenant dans le cadre d'une situation-type qui lui semble défavorable, celle du programme.

Dans notre cas, celui de la conception en architecture quelles difficultés a-t-on pu identifier dans les possibilités d'un usage ouvert des dispositifs numériques ? J'en soulignerai deux qui sont des conséquences directes de la définition de la situation-type "programme" mais qui offrent justement des moyens de la subvertir.

La première difficulté est liée à la pré-détermination des actions programmées.

Remarquons tout d'abord que les systèmes d'IA (Chomsky) ont bel et bien pénétrés les pratiques de conception mais que ces systèmes se développent sous une forme accessoire, ou additionnelle, qui n'est pas directement prescriptive pour l'usager, on peut parler alors d'un principe de conception augmentée. Pour la figuration par exemple, de tels systèmes peuvent inférer des informations géométriques pertinentes implicites dans le dessin et puis les afficher pour les montrer au sujet-concepteur. Ils augmentent ainsi le nombre d'informations accessibles par le dessinateur. C'est une réalité bien connue des infographistes. Car on rencontre depuis longtemps des modules inférenciels dans la plupart des logiciels graphiques. Ceux-ci ont été rendus populaires par le logiciel Sketchup. Le dessin y est prolongé par des traitements automatisés intégrés aux machines logicielles.

Mais le problème est que le sujet-concepteur s'oriente alors vers des activités de contrôle et de commande. Pour le dessin, par exemple, l'action de conception consiste alors moins en des gestes graphiques tangibles qu'en l'organisation de séquences indéterminées d'opérations qui, elles, sont déterminées puisque automatiques.

C'est là par exemple l'une des caractéristiques de ce que l'on nomme l'architecture paramétrique. Dans ce domaine on peut constater l'un des résultats pratiquement direct de l'application de la conception générative dans le domaine de l'architecture.

On en trouvera un exemple connu avec le module de design génératif intégré à l'application Grasshopper du logiciel Rhino qui s'appelle Galapagos et permet de lancer

***** Simondon, G., (1989). Du mode d'existence des objets techniques, Ed. Aubier, Paris. p.11 
des optimisations de contraintes formelles (allocations optimales de meubles dans une cuisine, de fenêtre dans une parois etc.)

Encore une fois, les solutions générées par le calcul ne se présentent pas comme des prescriptions mais comme une mise à disposition d'informations non triviales, des expertises, à destination du sujet-concepteur dans le cours de son action.

Une deuxième difficulté des migrations possibles de posture dans les contextes numériques de conception est liée à la notion de pré-définition des entités manipulées (les faits-informations). On sait, par principe, que tout programme exige une précision et une explicitation des données qu'il traite comme des opérations qu'il déclenche. Cela conduit à agir, dans notre situation-type programme, sur des objets respectant un format préalable, un format qui permette de suffisamment les détailler, les expliciter, les définir (ce sont des "faits structurés" ou opérationnels selon l'expression de Dewey).

A partir de là, les pratiques de conception et de figuration en architecture tendent vers un usage de représentations possédant une définition maximum, une haute définition. Cela débouche par exemple sur la manipulation de modèles à l'échelle un pour un, c'est à dire de maquettes numériques au sens du BIM.

Une telle maquette digitale peut être vue en effet comme une explicitation numérique totale de la représentation d'un projet. C'est un modèle complet, instancié ou non, c'est un prototype numérique conforme à un format standard de donnée (IFC).

Ainsi, pour résumer, les difficultés des conditions d'assistance numérique du travail de conception (en situation-type programme) résultent d'au moins deux causes. D'une part le déport d'actions élémentaires grâce à leur automatisation (exemple des modules d'inférence, des modules génératifs) et d'autre part la prédéfinition complète par explicitation des objets disponibles (exemple du format IFC).

Partant de ce constat, on peut dire que pour que le travail de "conception en contexte informatique" puisse rendre possible une migration de la posture ingénieur vers la posture praticien-réflexif il doit prendre pour point de départ l'intégration complète des deux contraintes méthodologiques que nous venons d'énoncer. En effet pour réaliser cette migration le sujet-concepteur doit accepter au moins deux accommodations de ses actions :

- d'une part (première accomodation) il doit s'approprier en pratique (éventuellement par le geste corporel) les automatismes disponibles dans les dispositifs de dessin augmenté. Et parvenir à faire de leurs usage une véritable praxis. On trouvera sur les platesformes d'auto-formation d'innombrables vidéos de démonstration qui illustrent ce genre d'appropriation par lequel le sujet-concepteur mobilise tout un savoir-faire relevant de la géométrie euclidienne dans l'espace, je renvoie par exemple aux démonstrations d'usage des modules inférenciels en cours de dessin pour le logiciel SketchUp.

- d'autre part (deuxième accommodation) le sujet-concepteur doit créer des marges d'indétermination dans sa manipulation des objets représentationnels de haute définition (type maquette numérique). Il s'agit d'accepter le principe de précision totale préalable des objets et des composants manipulés mais simultanément de maintenir le principe d'indétermination des usages, comme l'exige le principe de la machine ouverte chez Simondon. 
Cette indétermination ne peut pas concerner les actions élémentaires à mener puisqu'elles sont prédéfinies par le programme mais elle peut néanmoins concerner l'organisation stratégique des séquences et combinaisons de ces actions possibles. On passe alors de l'art de la fabrication à l'art de l'agencement.

Dans ce cas, nous pouvons parfaitement transposer en matière de conception numérique le même schéma de recherche, d'exploration et d'expérimentation que définit le maquettage de tradition en architecture (le travail en maquette). L'usage de la maquette, dans l'histoire de la conception architecturale, a toujours eu en effet à affronter cette double contrainte. La conception par production de prototypes mais également d'artefacts en séries, de variantes, de variations et d'agencements est inhérent à la conception par maquettage $e^{+t \dagger \dagger \dagger}$. Or ce schéma est transposable aux différents contextes numériques.

Rien ne s'oppose alors à ce que le sujet-concepteur adopte ce principe pragmatiste énoncé par Gilles Deleuze à propos de la création artistique lorsqu'il écrit : "il faut être précis et indéterminé". Les augmentations de capacité de calcul n'invalident pas cette possibilité, mais observons que ces systèmes partiellement automatiques doivent être utilisés comme des machines incitatives (ouvertes), c'est à dire des machines qui n'abaissent pas les compétences du concepteur en matière de stratégie de conception. Elles ne doivent pas déqualifier le concepteur mais au contraire être appropriables par lui de telle sorte qu'il puisse augmenter son savoir-faire de métier.

Les conséquences de cette migration (élémentaire) de la posture ingénieur vers la posture praticien-réflexif en contexte numérique sont importantes pour la conception. Apparemment, elles peuvent correspondre à des ruptures culturelles dans le domaine de l'enseignement notamment. Car si la pratique-réflexive de conception peut prendre pour point de départ des objets entièrement prédéfinis (explicites) et manipuler des actions élémentaires déterminées c'est à dire automatisées alors l'idée reçue (académique, scolastique) d'une conception architecturale linéaire et cartésienne allant du flou vers le précis est remise en cause pratiquement.

Elle est remise en cause au profit d'une conception divergente qui s'appuie sur la combinatoire et agit par création de configuration. Une conception divergente capable de produire des "agencements" en opposition à la conception convergente propre à la rationalité technique traditionnelle (celle de l'homo faber) qui produirait des objets. Il existe de nombreux architectes qui revendiquent aujourd'hui ces possibilités de conception divergentes. Ce sont des conceptions non-formelles qui se développent pourtant en contexte numérique ou hybride. Il devient possible d'y déployer des méthodes structuralistes au sens que donne à ce terme dans plusieurs de leur travaux un certain nombre d'architectes contemporains comme Anne Lacaton, et Jean-Philippe Vassal par exemple dans leur projet pour une salle polyvalente à Lille qui se présente comme une machine incitative à destination des usagers de la salle.

Pour conclure cette intervention je veux souligner que les outils programmatiques numériques intégrés à une pratique réflexive fournissent donc l'occasion de penser et de pratiquer la conception en dehors du mythe de la création ex-nihilo et du contrôle a priori des projets (posture de l'ingénieur). Dans ce cas, le travail de conception par mise au point

†† $d^{\prime}$ Aujourd'hui, $n^{\circ} 404$, Paris. https://issuu.com/daniel-estevez/docs/aa_larchitecture_daujourdhui_de_cop 
et variations prend pour point de départ un état prédéfini et donc pré-existant de l'artefact à concevoir et puis agit ensuite par transformation de ce précédent. La combinatoire des actions demeure alors le véritable terrain exploratoire pour le sujet-concepteur dans sa recherche.

Comme je viens de tenter de le montrer, ces démarches qui résultent de la migration de posture ingénieur-praticien permettent l'actualisation des approches dites structuralistes de la conception architecturale. Cette dimension structuraliste de la migration ingénieurpraticien nous introduirait finalement dans une nouvelle situation-type, celle du projet. Je ne présenterai pas aujourd'hui les autres migrations telles que nous avons pu les observer au cours de nos expériences didactiques. Elle feront l'objet de différentes publications qui sont en préparation sur ce sujet.

Je voudrais enfin conclure cet exposé en évoquant toutes les personnalités, les chercheurs, les auteurs, les doctorants, les subjectivités qui font et nourrissent ces recherches en cours et en particulier ceux qui ont inspiré les chapitres que je n'ai pas abordés aujourd'hui. Comme dans toute recherche, ces visages amis forment une constellation de travail. Toute recherche est une construction personnelle mais elle est produite par créolisation comme le disait Edouard Glissant, et résulte peut-être également de nos propres capacités de migrations. 